Gründen mit wesentlich höheren Konzentrationen $(8 \%)$ gearbeitet wurde, die durch Interferenzeinfluß die Meßwerte etwas erniedrigen.

Die ermittelten Längen (jetzt $237-240$, früher $230-240 \AA$ ) stimmen überein. Ebenso ergibt sich übereinstimmend ein elliptischer Zylinder als beste Interpretation der Streukurve. Seinerzeit wurde ein $R_{\mathrm{q}}=14,5 \AA$ ermittelt (mit den Achsen 9,5 und $28 \AA$ ), ein Wert, der etwas größer ist als $R_{\mathrm{q}, \mathrm{K}}=13,6$ (mit den Achsen 10.9 und $25 \AA$ ), also der Streumassenradius des Kernanteils, auf den $76 \%$ der Masse entfallen. Die Zweiteilung in Kern- und Hüllenanteil konnte damals nicht festgestellt werden. Abgesehen von der Möglichkeit, daß durch die hohe, damals angewendete Konzentration die Verhältnisse etwas modifiziert sind und auch die seinerzeit erzielte Genauigkeit die heute gegebene nicht erreicht, könn- ten Unterschiede im Präparat vorgelegen haben, nachdem die Herstellung in der Zwischenzeit modifiziert wurde ${ }^{2 a}$. Neuerdings stand uns ein nach einem chromatographischen Verfahren ${ }^{2 \mathrm{a}, 3}$ besonders gereinigtes Präparat zur Verfügung. Wir möchten jedenfalls dem neueren Ergebnis den Vorzug geben und die Vorstellung des Kern-Hülle-Modells als die derzeit beste Interpretation der Messungen betrachten.

Herrn Professor Schultze (Behring-Werke Marburg/Lahn) möchten wir für die Überlassung der Präparate und wichtige Hinweise bei der Diskussion der Ergebnisse verbindlichst danken.

Die Rockef eller Foundation hat die Untersuchung durch Bereitstellung von apparativen Mitteln unterstützt, wofür ebenfalls bestens gedankt sei.

\title{
Charakterisierung der verschiedenen, nach Infektion mit Newcastle Disease Virus auftretenden, nichtinfektiösen-hämagglutinierenden Teildhen
}

\author{
Von Rudolf Rott, Ismail M. Reda* und Werner Schäfer \\ Aus dem Max-Planck-Institut für Virusforschung, Tübingen \\ (Z. Naturforschg. 18 b, 188-194 [1963] ; eingegangen am 23. November 1962)
}

Herrn Prof. A. Butenand zum 60. Geburtstag gewidmet

\begin{abstract}
Ebenso wie bei den Influenzaviren treten auch beim NDV zwei Arten von nichtinfektiösen-hämagglutinierenden Teilchen auf, die als "Inkomplette Formen" und "Viromikrosomen" bezeichnet werden.
\end{abstract}

Untersuchungen an Influenzaviren haben ergeben, daß hier mindestens zwei Arten von nichtinfektiösenhämagglutinierenden Teilchen $(\mathrm{NIH})$ vorkommen ${ }^{1}$.

Die sogenannten „Inkompletten Formen" 2 werden von den Wirtszellen in das umgebende Medium ausgeschieden. Ihre Oberfläche gleicht der der infektiösen Partikel (Viria); wie bei diesen, ist sie auch hier mit feinen, stachelartigen Gebilden besetzt, die Träger der hämagglutinierenden Aktivität sind ${ }^{3,4}$. Die Inkompletten Formen unterscheiden sich aber dadurch grundlegend von den Viria, daß sie nicht die volle Ausstattung an virusspezifischem, RNShaltigen. genetischen Material (g-Antigen) in ihrem Innern enthalten ${ }^{1.5 .6}$. Inkomplette Formen werden

* Universität Kairo, V.A.R.

1 R. Rotr u. W. SсHÄfer, Z. Naturforschg. 16 b, 310 [1961].

2 P. v. Magnus, Advances in Virus Res. 2, 59 [1954].

3 A. P. Waterson, R. Rott u. W. Schäfer, Z. Naturforschg. 16 b, 154 [1961]. von den Wirtszellen vor allem dann ausgeschieden, wenn man das Virusmaterial in sehr hohen Dosen passagiert (v. Magnus-PhäNomen) 2 .

Die zweite Art von NIH der Influenzaviren, die „Viromikrosomen“ "1, ist regelmäßig in Homogenaten infizierter Zellen enthalten. Durch Änderung der Infektionsbedingungen läßt sich ihre Menge nicht merklich erhöhen. Diese aus dem Innern der Wirtszellen herstammenden NIH gleichen, wie schon aus ihrer Bezeichnung hervorgeht, in ihrem morphologischen und biochemischen Verhalten den Mikrosomen normaler Zellen ${ }^{1}$. Das in ihnen vorliegende Hämagglutinin ist nicht in Form stachelartiger Gebilde an der Oberfläche angeordnet, sondern scheint

\footnotetext{
C. Morgan, K. C. Hsu u. H. M. Rose, J. exp. Medicine 116, 553 [1962].

5 G. L. Ada u. B. T. Perry, J. gen. Microbiol. 14, 623 [1956].

${ }^{6}$ F. S. Lief u. W. Hexle, Virology 2, 782 [1956].
} 
vielmehr in den membran-artigen Strukturen enthalten zu sein. Eine dem g-Antigen entsprechende Komponente konnte in den Viromikrosomen nicht nachgewiesen werden.

Auf Grund der bisher bei den Influenzaviren vorliegenden Untersuchungsergebnisse wird angenommen, daß die Inkompletten Formen Viruspartikel sind, in die während der Fertigstellung an der Zellperipherie keine ausreichende Menge von g-Antigen eingebaut wurde. Demgegenüber scheinen die Viromikrosomen Bestandteile des endoplasmatischen Retikulums infizierter Zellen zu sein, in denen das Material der Hämagglutinin-Stacheln fertiggestellt wurde und die danach mit diesem beladen sind.

Beim Newcastle Disease Virus (NDV), einer anderen RNS-haltigen Virusart, die dem Mumpsvirus und den Parainfluenzaviren nahesteht, wurden NIH zunächst von Granoff und Mitarb. ${ }^{7-9}$ vor allem in Homogenaten infizierter Zellen gefunden. Später konnten von unserer Arbeitsgruppe NIH auch bei diesem Virus in weitgehend reiner Form aus der die Wirtszellen umgebenden Flüssigkeit (Eiflüssigkeit) isoliert werden ${ }^{10}$. Nach vorläufigen elektronenoptischen Befunden scheinen diese morphologisch den Inkompletten Formen der Influenzaviren zu entsprechen. Es lag deshalb die Annahme nahe, daß bei zwei derartig verschiedenen Virusarten, wie den Influenzaviren und dem NDV, im Hinblick auf die NIH analoge Verhältnisse vorliegen.

Um hierüber Klarheit zu gewinnen, wurden nunmehr auch die NIH des NDV eingehender charakterisiert und mit dem ND-Virion verglichen. Die aus Eiflüssigkeit stammenden NIH werden im folgenden zunächst als freie, nichtinfektiöse-hämagglutinierende Teilchen (FHA), die aus Zellhomogenaten isolierten als zellgebundene, nichtinfektiöse-hämagglutinierende Teilchen (ZGHA) bezeichnet.

\section{Material und Methoden}

Eine Reihe der bei den Versuchen angewendeten Methoden wurde schon in früheren Veröffentlichungen beschrieben, auf die in diesem Zusammenhang verwiesen sei ${ }^{1,11}$. Dies gilt für die Hämagglutinations-(HA), Infektiositäts- und Komplementbindungs-(KB)-Teste sowie für die Bestimmung der Neuraminidase-Aktivität.

7 A. Granoff, O. C. Liu u. W. Henle, Proc. Soc. exp. Biol. Med. 75, 684 [1950].

8 A. Granoff u. W. Henle, J. Immunology 72, 329 [1954].

9 A. Granoff, Virology 1, 516 [1955].

10 R. Rотt, I. M. Reda u. W. Sсhäfer, Virology 16, 207 [1962].

11 F. M. Davenport, R. Rott u. W. Sсhäfer, J. exp. Medicine 112, 765 [1960].
1. Virusstäm me

Für die Untersuchungen verwendeten wir den NDV. Stamm „Italien“ 12 und eine hitzestabile Mutante (C) des Stammes „Beaudette“ ${ }^{13}$. Das Material wurde durch Infektion von 11 Tage lang bebrüteten, befruchteten Hühnereiern mit $10^{2} I D_{50}$ gewonnen.

\section{Antis e rum}

Es wurde ein Rekonvaleszenten-Serum von Goldhamstern benutzt, die mit dem an diese Tierart adaptierten Stamm „Italien“ infiziert waren ${ }^{14}$.

3. Bestimmung der hämolytischen und interferierenden Aktivität

Zur Bestimmung der hämolytischen Aktivität wurden gereinigte Präparate von Viria, FHA und ZGHA auf gleichen HA-Titer eingestellt, über Nacht gegen Aqua dest. und danach 4 Stdn. gegen 3,8-proz. Na-Citratlösung dialysiert. Die Präparate wurden anschließend nach Potenzen von 2 in Na-Citratlösung verdünnt und dann zu $1 \mathrm{~cm}^{3}$ jeder Verdünnungsstufe das gleiche Volumen einer 1-proz. Suspension von Hühnererythrocyten gegeben. Die Mischung wurde $3 \mathrm{Stdn}$. bei $37{ }^{\circ} \mathrm{C}$ inkubiert. Nach kurzem Zentrifugieren der Ansätze bei $2000 \mathrm{~g}$ wurde in den Überständen die Extinktion bei $544 \mathrm{~m} \mu$ im Spektrophotometer bestimmt. Als 100\%. Wert diente eine entsprechende Erythrocytensuspension, die in 0,01-proz. Saponinlösung aufgenommen war $^{8}$.

Bei den Interferenzversuchen wurde im Prinzip das von Paucker und Hente ${ }^{15}$ beschriebene Verfahren angewendet. Gereinigte Präparate von FHA, ZGHA und Viria wurden auf $256 \mathrm{HA}$-Einheiten $/ \mathrm{cm}^{3}$ eingestellt und unter den von Tokumaru und SChäFer ${ }^{16}$ angegebenen Bedingungen 2 Min. lang mit UV-Licht bestrahlt (eingestrahlte Energie: etwa $13 \mathrm{erg} / \mathrm{mm}^{2} / \mathrm{sec}$ ). In Vorversuchen war festgestellt worden, $\mathrm{da} \beta$ sich nach dieser Bestrahlungszeit infektiöse Partikel nicht mehr nachweisen lassen. $0,5 \mathrm{~cm}^{3}$ des uv-inaktivierten Materials wurden jeweils in die Allantoishöhle embryonierter Hühnereier injiziert, die 10 Tage lang bebrütet waren. 20 Stdn. später erfolgte die Superinfektion mit aktivem Virus. Es wurden dafür verschiedene Mengen verwendet; jeder einzelne Ansatz umfaßte 4 Eier. 30 Stdn. nach der Superinfektion wurde in der von den einzelnen Ansätzen gesammelten Eiflüssigkeit die Anzahl der infektiösen Einheiten mit Hilfe des Plaquetestes bestimmt. Bei den Kontrollen wurde an Stelle der uvinaktivierten Viruseinheiten gepufferte NaCl-Lösung $\left(p_{\mathrm{H}} 7,2\right)$ injiziert.

12 W. Schäfer, G. Schramm u. E. Traub, Z. Naturforschg. 4 b. 157 [1949]

13 A. Granof F, Virology 9, 636 [1959].

14 R. Rott u. W. Schäfer, Z. Naturforschg. 17 b, 160 [1962].

15 K. Paucker u. W. Henle, Virology 6, 198 [1958].

16 T. Tokumaru u. W. Schäfer, Z. Naturforschg. 13 b, 704 [1958]. 
4. Reinigung der NIH und Viria

FHA wurden aus der Eiflüssigkeit NDV-infizierter Hühnereier gewonnen. Sie wurden durch Adsorptionschromatographie, fraktioniertes Zentrifugieren und Adsorption-Elution an bzw. von Hühnererythrocyten nach einer schon früher angegebenen Methode gereinigt ${ }^{10}$.

Die $Z G H A$ wurden im Prinzip wie die entsprechenden Formen der Influenza aus Homogenaten infizierter Chorioallantoismembranen durch Adsorption-Elution an bzw. von Hühnererythrocyten und anschließendes fraktioniertes Zentrifugieren isoliert ${ }^{1}$. Vor Zugabe der Erythrocyten wurde das Gewebehomogenat 30 Min. bei $37^{\circ} \mathrm{C}$ bebrütet und durch Zentrifugieren bei pi 5 in der präparativen Ultrazentrifuge gereinigt. Die Adsorption an die Erythrocyten und das anschließende Waschen der Komplexe wurden bei $p_{\mathrm{H}} 8,5$ in der Kälte, die Elution des Virusmaterials bei $p_{H} 7,2$ bei Raumtemperatur durchgeführt (60 Min); hierfür wurde gepufferte Kochsalzlösung verwendet.

Die Viria wurden zunächst zusammen mit den FHA durch Chromatographie über Calciumphosphatsäulen vorgereinigt ${ }^{17}$ und von den FHA durch Ultrazentrifugieren bei pi 5 abgetrennt.

\section{Isolierung von Ribosomen}

Zur Isolierung der Ribosomen wurden die ZGHA, wie schon früher bei den entsprechenden Formen der Influenzaviren beschrieben, mit Trichlorfluoräthan („Frigen“, Farbwerke Hoechst) geschüttelt und durch Ultrazentrifugieren angereichert ${ }^{1}$.

\section{Schütteln mit Äther}

Es wurde das von $\mathrm{S}_{\text {CHÄFER }}$ und $\mathrm{Z}_{\text {ILLIG }}{ }^{18}$ beschriebene Verfahren angewendet.

$$
\text { 7. Chemische Analysen }
$$

Der Stickstoffgehalt wurde mit Hilfe der Mikromethode nach $\mathrm{K}$ jeld a h l ermittelt. Die Bestimmung der Ribonucleinsäure (RNS) erfolgte nach der von Hurlbert und Mitarbb. ${ }^{19}$ beschriebenen Methode.

\section{Physikalisch-chemische Methoden}

Für die Bestimmung der Sedimentations-Geschwindigkeiten wurde die analytische Ultrazentrifuge der Firma Beckman Instruments (Modell E) verwendet.

Die Zentrifugierung im CsCl-Dichtegradienten erfolgte im Schwingrotor SW 39 der präparativen SpincoUltrazentrifuge (L). Proben von jeweils 10 Tropfen wurden aus den Zelluloseröhrchen entnommen und die Brechungsindices im Refraktometer der Firma Carl Zeiss bestimmt.

\section{Elektronenoptische Untersuchung}

Die bedampften (Pt, Rh; 25 $)$ Präparate wurden im EM 8 (Zeiss, AEG), die mit Phosphorwolframsäure

17 I. M. Reda u. R. Rott, Zbl. Vet. Med. IX, 158 [1962].

18 W. Schäfer u. W. Zillig, Z. Naturforschg. 9 b, 779 [1954].

19 R. B. Hurlbert, H. Schmitz, A. F. Brumm u. R. v. Potter, J. biol. Chemistry 209, 23 [1954].
(PWS) kontrastierten im Elmiskop I der Firma Siemens untersucht. Bei der letztgenannten Technik verwendeten wir das von Brenner und Horne ${ }^{20}$ angegebene Verfahren.

\section{Ergebnisse}

1. Vorkommen von NIH bei zwei verschiedenen Stämmen des NDV

Zunächst wurden bei unseren Untersuchungen zwei NDV-Stämme verwendet, die sich in ihrer Pathogenität gegenüber Hühnern grundlegend unterscheiden. Der Stamm „Italien“ ist hoch, der Stamm "Beaudette" dagegen gering pathogen. Es zeigte sich, daß beim Stamm „Beaudette“ größere Mengen von FHA in das umgebende Medium ausgeschieden werden als beim Stamm „Italien“. Im ersten Fall waren im Durchschnitt etwa $10-20 \%$ der in der Eiflüssigkeit vorhandenen Virusteilchen NIH, im letzteren dagegen nur 0,5-2,5 Prozent. Die Ausbeute an ZGHA war dagegen bei beiden etwa gleich groß. Auf Grund dieser Befunde wurde für die weiteren Experimente ausschließlich der Stamm „Beaudette" verwendet.

\section{Biologische Eigenschaften der NIH}

Das Virion der ND besitzt neben der infektiösen und hämagglutinierenden Eigenschaft noch virusspezifische antigene Aktivität. Es ist Träger eines Enzyms, das den Charakter einer Neuraminidase hat und enthält außerdem ein hämolytisches Prinzip, das nach neueren Untersuchungen von der Neuraminidase abgetrennt werden kann ${ }^{21}$. Über seine Natur besteht noch keine Klarheit.

Die Infektiosität der isolierten FHA- und ZGHAPräparate war regelmäßig sehr gering. Das Verhältnis von plaquebildenden $(\mathrm{PBE})$ zu hämagglutinierenden (HAE) Einheiten ( $\log \mathrm{PBE} / \log \mathrm{HAE}$ ) schwankte bei den FHA-Präparaten zwischen 1,9 und 3,7, bei den ZGHA-Konzentraten zwischen 2,8 und 4,2. Bei gereinigten Konzentraten der Viria lag es dagegen im Bereich von 6.8 bis 7,1. Bezogen auf hämagglutinierende Einheiten war also die Infektiosität der ZGHA- und FHA-Präparate in der Regel mindestens 1000 -fach geringer als die der gereinigten Konzentrate von Viria. Da die Ausgangsmate-

20 S. Brenner u. R. W. Horne, Biochim. biophysica Acta [Amsterdam] 34, 103 [1959].

21 G. Rebel, R. Fontages u. L. Colobert, Ann. Inst. Pasteur 102, 137 [1962]. 
rialien für die Isolierung beider NIH-Formen Viria enthielten, ist anzunehmen, daß die in den entsprechenden gereinigten Präparationen enthaltenen infektiösen Einheiten restliche Viria waren.

Im Gegensatz zur infektiösen Aktivität liegen die hämagglutinierende und die durch die Komplementbindungs-Reaktion erfaßbare antigene Aktivität aller drei Teilchenarten, wenn man diese Aktivitäten auf Stickstoffgehalt bzw. HA-Einheiten bezieht, in der gleichen Größenordnung (Tab. 1). Dies trifft

\begin{tabular}{|l|c|c|c|c|}
\hline Material & $\begin{array}{c}\text { N/HAE } \\
{\left[\cdot 10^{-8} \mathrm{~g}\right]}\end{array}$ & $\begin{array}{c}\text { PBE/ } \\
\mathrm{HAE} \\
{\left[\log _{10}\right]}\end{array}$ & $\begin{array}{l}\text { KBE/ } \\
\text { HAE }\end{array}$ & $\begin{array}{c}\text { Neura- } \\
\text { minidase- } \\
\text { E/HAE }\end{array}$ \\
\hline FHA & $0,3-1,0$ & $1,9-3,7$ & 0,008 & 0,011 \\
ZGHA & 5,7 & $2,8-4,2$ & 0,006 & 0,010 \\
Viria & 4,2 & $6,8-7,1$ & 0,006 & 0,013 \\
\hline
\end{tabular}

Tab. 1. Biologische Eigenschaften der NIH und des Virion der Newcastle Disease.

auch für die Neuraminidase-Aktivität zu (s. Tab. 1), während die hämolytische Aktivität bei FHA und ZGHA eindeutig geringer ist als bei den Viria (Abb. 1). Im Hinblick auf die ZGHA war dies nach den Untersuchungen von Granoff und $\operatorname{Henle}^{8}$ zu erwarten.

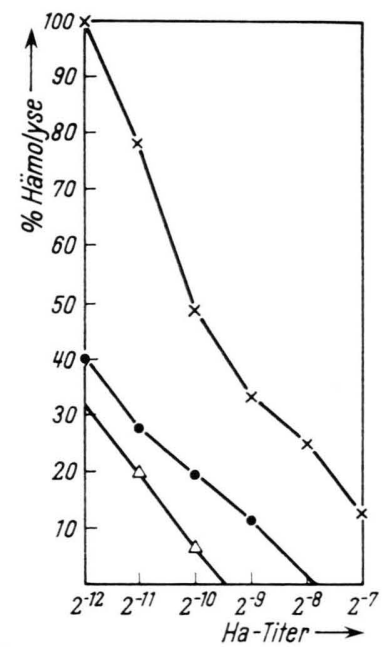

Abb. 1. Hämolyse-Aktivitäten der NIH und des Virion der Newcastle Disease. $\times$ Virion, $\bullet$ FHA, $\triangle$ ZGHA.

Wie bei den Influenzaviren läßt sich auch beim NDV mit uv-inaktivierten Viria eine homologe Interferenz erzeugen ${ }^{22}$. Von den NIH besitzen diese Fähigkeit nach früheren Untersuchungen bei den Influenzaviren ${ }^{1,15}$ wohl die Inkompletten Formen, nicht aber die Viromikrosomen. Die nunmehr durchgeführten Interferenzversuche mit NDV ergaben 22 W. HENLE, J. Immunology 64, 203 [1950]. demgegenüber, daß weder die ZGHA noch die FHA dieses Virus eine nachweisbare interferierende Aktivität besitzen. Das Ergebnis eines dieser Versuche ist in Abb. 2 wiedergegeben.

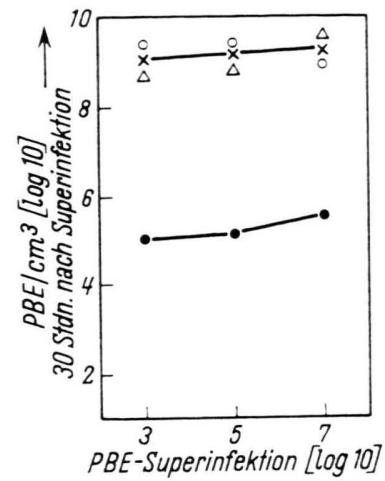

Abb. 2. Interferierende Aktivitäten uv-bestrahlter Viria und NIH der Newcastle Disease. $\bullet$ Virion, $\triangle$ FHA, ○ ZGHA, $\times \mathrm{NaCl}$.

3. Chemische Eigenschaften

Wie schon eingangs erwähnt, enthalten die Inkompletten Formen der Influenza im Vergleich zu den Viria eine geringere Menge an RNS-haltiger Innenkomponente (g-Antigen). Analog scheinen die Verhältnisse bei den FHA des NDV zu liegen. Eine vergleichende quantitative Bestimmung der RNS in gereinigten Präparationen von Viria und FHA der ND ergab, daß die letzteren im Vergleich zu den infektiösen Partikeln praktisch keine Ribonucleinsäure enthalten. Das für diesen Versuch verwendete Material wurde während der Reinigungsprozedur mit RNase behandelt $\left(200 \mathrm{\gamma} / \mathrm{cm}^{3}\right.$, über Nacht bei Raumtemperatur), um virusfremde RNS, die an die Einheiten adsorbiert sein könnte, zu entfernen. 8000 HA-Einheiten der Präparate enthielten bei den Viria $90 \gamma$ RNS, bei den FHA weniger als $0,1 \gamma$ RNS.

Da damit gerechnet werden mußte, daß die ZGHA größere Mengen von zelleigener RNS enthalten, wurde hier auf eine quantitative Bestimmung der RNS verzichtet. Durch Behandeln mit Frigen konnten aber aus diesen Teilchen, ebenso wie aus den Viromikrosomen der Influenzaviren, Partikel herausgelöst werden, die den Ribosomen der normalen Zellen entsprechen. Nach der elektronenoptischen Untersuchung beträgt ihre Größe etwa $15 \mathrm{~m} \mu$, nach der UV-Absorption enthalten sie etwa $46 \%$ Nucleinsäure $\left(E_{260} / E_{280}=1,87\right)$. Die chemische Analyse ergab, daß es sich ausschließlich um RNS handelt.

Frühere Untersuchungen hatten Hinweise dafür geliefert (l. c. ${ }^{1}$ und unveröffentlichte Befunde), daß 
Virusteilchen, die in ihrer Hülle Lipoproteide der peripheren Membran der Wirtszellen enthalten, sich gegenüber $\ddot{A}$ ther anders verhalten als solche, deren Lipoprotein-Komponente aus dem endoplasmatischen Retikulum stammt. Im ersten Falle kann durch Schütteln mit Äther bei $37^{\circ} \mathrm{C}$ das virusspezifische Hämagglutinin nahezu quantitativ herausgelöst und in die Wasserphase überführt werden. Im zweiten Falle wird, wenn überhaupt, nur ein geringer Prozentsatz des Hämagglutinins in der Wasserphase gefunden. Wie Tab. 2 zeigt, verhalten sich Viria und

\begin{tabular}{|c|r|r|r|}
\hline $\begin{array}{c}\text { Äther- } \\
\text { behandlung }\end{array}$ & Viria & FHA & ZGHA \\
\hline vor & 8000 & 8000 & 8000 \\
nach & 32000 & 21000 & $<8$ \\
\hline
\end{tabular}

Tab. 2. Verhalten der NIH und des Virion der Newcastle Disease gegenüber Äther. Menge der $\mathrm{HAE} / \mathrm{cm}^{3}$ nach Ätherbehandlung in der Wasserphase.

FHA des NDV bei Ätherbehandlung wie die mit peripherem Membranmaterial versehenen Partikel, wogegen die ZGHA in dieser Hinsicht den mit cytoplasmatischem Material ausgestatteten Einheiten gleichen.

Das unterschiedliche Verhalten beider NIH-Formen gegenüber Äther sowie die Tatsache, daß die ZGHA ribosomenähnliche Teilchen enthalten, während in den FHA praktisch keine RNS nachzuweisen ist, sprechen dafür, daß beide trotz ihres weitgehend gleichartigen biologischen Verhaltens zwei grundsätzlich verschiedene Arten von nichtinfektiösen-hämagglutinierenden Formen repräsentieren.

\section{Physikalische Eigenschaften}

Für die physikalische Charakterisierung der NIH des NDV wurden die Ultrazentrifugierung und die elektronenmikroskopische Untersuchung herangezogen.

In der analytischen Ultrazentrifuge sedimentieren die infektiösen Partikel mit einer $s_{20}$ von etwa $1100 \mathrm{~S}$ (s. Tab. 3, vgl. l. c. ${ }^{12}$ ). Die Sedimentations-Konstanten der NIH sind wesentlich geringer (s. Tab. 3); bei den FHA schwanken die Werte zwischen 200 und $250 \mathrm{~S}$, bei den ZGHA zwischen 90 und $300 \mathrm{~S}$.

Entsprechende Unterschiede kamen auch bei der Zentrifugierung im CsCl-Dichtegradienten zum Ausdruck (s. Abb. 3). Die Viria wurden in der Hauptsache in einer Fraktion mit einer Dichte von 1,280

22 R. H. Horne, A. P. W Wterson, P. Wildy u. A. E. Farnham, Virology 11, 79 [1960].

\begin{tabular}{|c|c|c|c|}
\hline \multirow{2}{*}{ Material } & \multirow{2}{*}{ PBE/HAE } & \multicolumn{2}{c|}{$s_{20}$} \\
\cline { 3 - 4 } & & optisch & biologisch* \\
\hline \multirow{2}{*}{ Virion } & 6,9 & 1100 & 1087 \\
& 7,0 & 1185 & - \\
\hline FHA & 2,9 & 257 & 233 \\
& 2,5 & 214 & - \\
& 2,2 & 205 & 215 \\
& 2,3 & 206 & - \\
& 3,7 & 224 & 230 \\
& 2,1 & 204 & - \\
ZGHA & 2,3 & 211 & - \\
& & & - \\
& 3,8 & 137 & - \\
& 2,9 & 221 & - \\
& 4,2 & 91 & - \\
& 3,4 & 98 & 123 \\
\hline & 2,7 & 291 & \\
& 3,1 & 100 & \\
\hline
\end{tabular}

Tab. 3. Sedimentationskonstanten der NIH und des Virion der Newcastle Disease. ${ }^{*} s_{20}$ wurde biologisch aus dem Abfall der HA nach Ultrazentrifugierung bestimmt ${ }^{10}$.

$(n=1,3550)$ gefunden, die FHA in einer solchen mit einer Dichte von 1,262 $(n=1,3530)$. Die Dichte der ZGHA streut stärker; sie liegt zwischen 1,202 und $1,242(n=1,3460-1,3505)$.

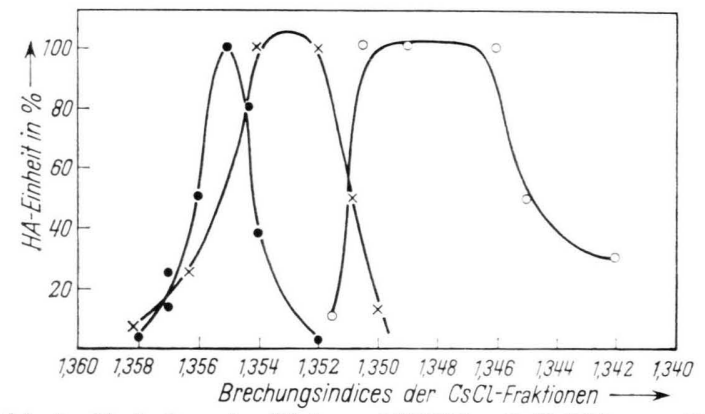

Abb. 3. Verhalten der Viria und NIH im CsCl-Dichtegradienten. - Virion, $\times$ FHA, ○ ZGHA.

Weitergehende Aufschlüsse lieferte die elektronenmikroskopische Untersuchung. Bei den Präparaten von Viria sieht man Teilchen mit einem Durchmesser von $130-180 \mathrm{~m} \mu$, die auf Grund der Untersuchungen mit PWS eine mit $100 \AA$ langen Stacheln versehene Hülle besitzen ${ }^{22 a}$. Im Innern enthalten sie eine Struktur, die in ihrem Feinbau an das Tabakmosaikvirus erinnert. Sie ist Träger der Virus-RNS ${ }^{23,24}$.

Die FHA (Abb. 4a*, b) sind im allgemeinen kleiner, ihr Durchmesser schwankt zwischen 40 und $120 \mathrm{~m} \mu$. Die größeren weisen häufig eine zentrale Eindellung auf (Abb. 4b). Vereinzelt sind auch

23 W. Sснӓғеr U. R. Rott, Z. Naturforschg. 14 b. 629 [1959].

24 R. Rott u. W. Schäfer, Virology 14, 298 [1961].

* Abb. 4 s. Tafel S. 192 a. 


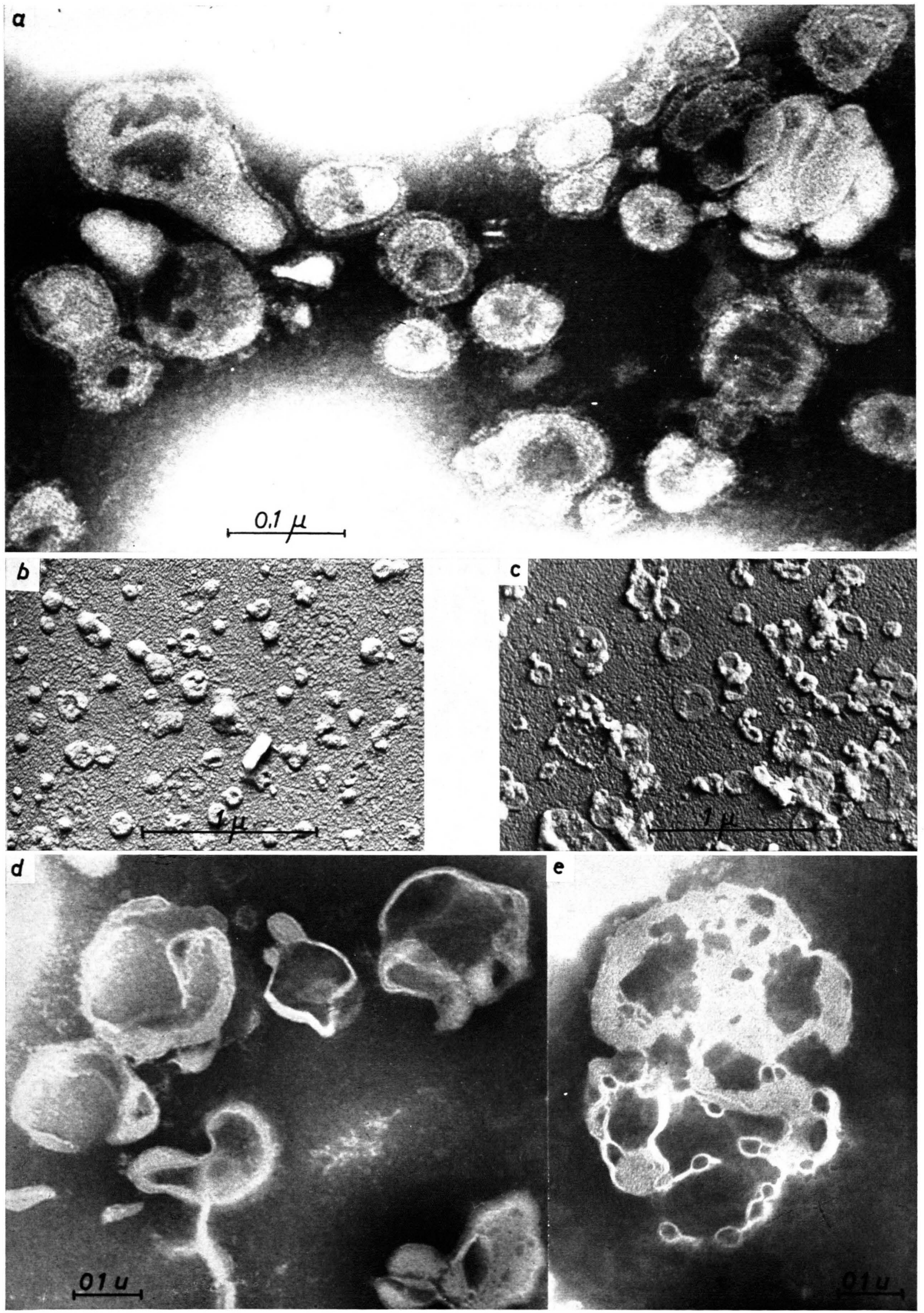

Abb. 4. Flektronenmikro-kopi-che Aufnahmen. a) FHA (PIS). b) FHA (bedampft). c) 7CHA (bedampft). 


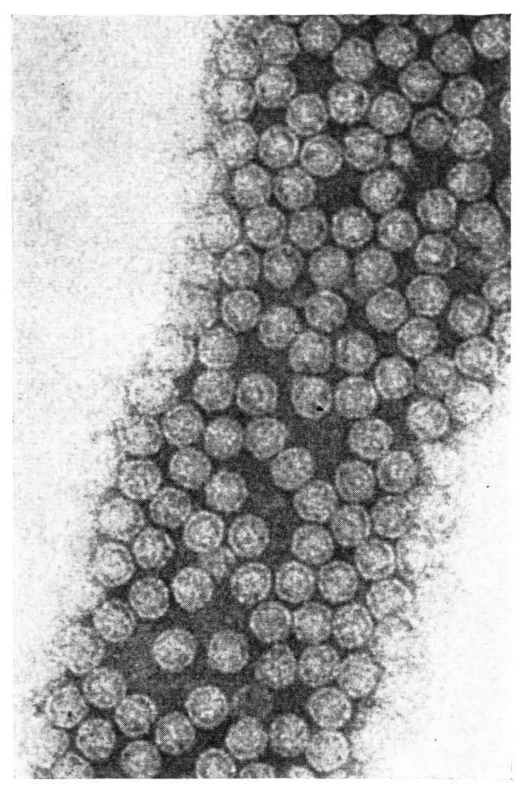

Fig. 1. Electron micrograph of negatively stained M 12 phages; magnification $160000 \times$.

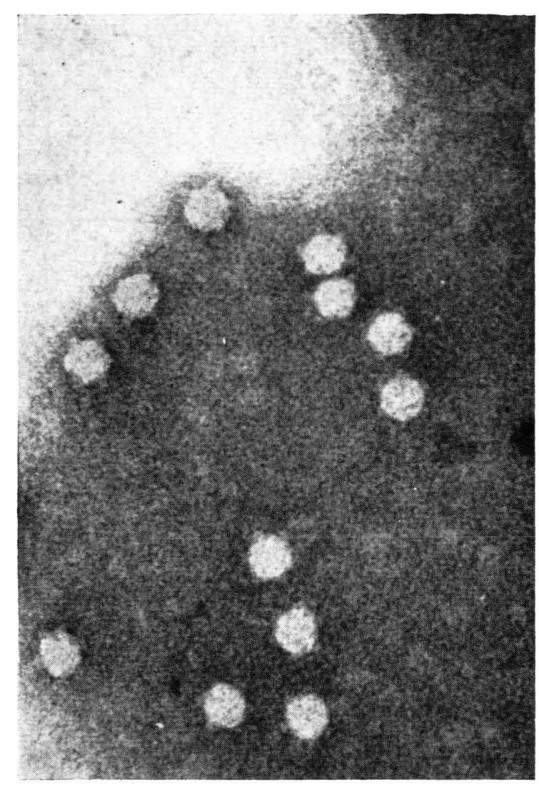

Fig. 2. Electron micrograph of negatively stained M 20 phages; magnification $160000 \times$.

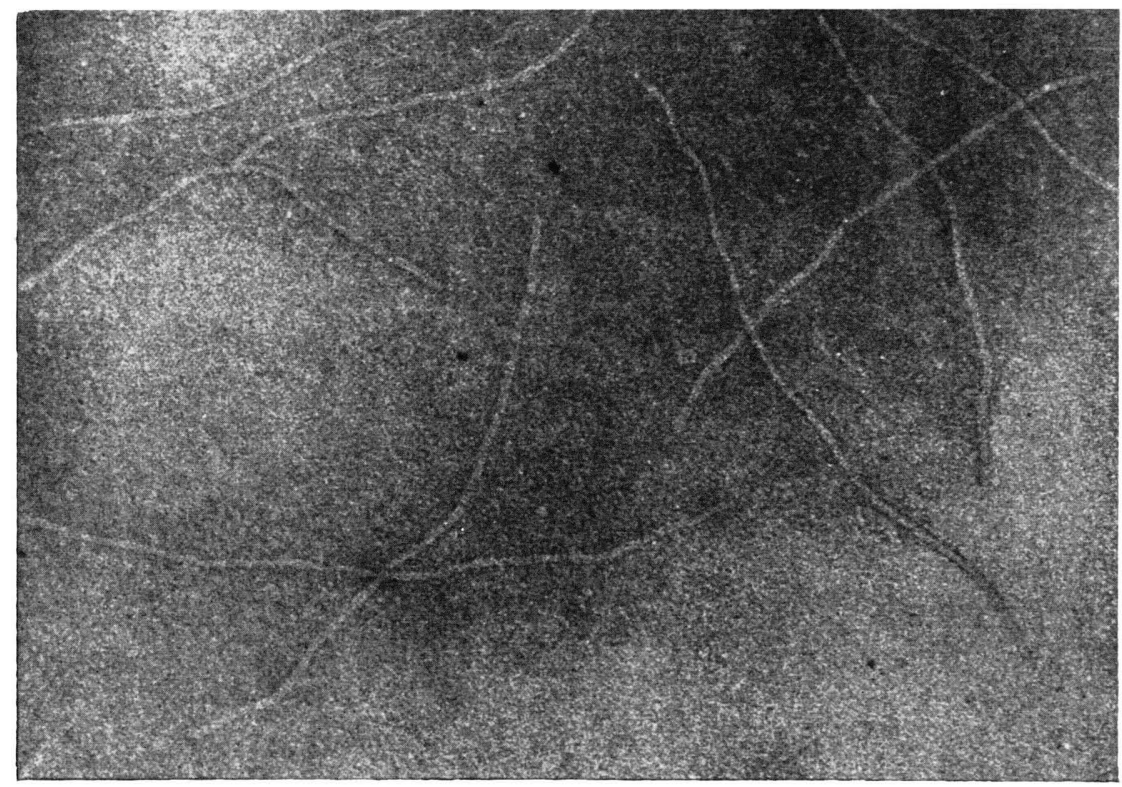

Fig. 3. Electron micrograph of negatively stained M 13 phages; magnification $80000 \times$. 
hantelförmige Teilchen zu sehen. Nach Kontrastierung mit PWS ist bei den FHA eine an das Tabakmosaikvirus erinnernde Innenstruktur nicht zu erkennen. Bei ihnen wird vielmehr häufig Kontrastmittel im Zentrum gefunden; es ist deshalb anzunehmen, daß sie keine Innenkomponente enthalten. Dies war zu erwarten, weil bei diesen Teilchen keine RNS gefunden wurde. In der Feinstruktur ihrer Hülle unterscheiden sich die FHA aber elektronenoptisch nicht von den Viria. Wie bei diesen, so ist sie auch bei ihnen mit feinen, $100 \AA$ langen und $35 \AA$ starken, stachelartigen Gebilden besetzt.

Bei den ZGHA (Abb. $4 \mathrm{c}-\mathrm{e})$ ließen sich weder derartige Stacheln noch die Innenkomponente der Viria nachweisen. Bei ihnen handelt es sich um vielgestaltige, blasenartige Formen, deren Membran eine lamellenartige Struktur zu besitzen scheint (s. Abb. 4e). Die Größe dieser Teilchen schwankt zwischen 60 und $550 \mathrm{~m} \mu$. Sie sind elektronenoptisch den Teilchen einer Mikrosomenfraktion aus normalen Chorioallantois-Membranen sehr ähnlich (vgl. l. c. ${ }^{1}$ ).

\section{Besprechung der Ergebnisse}

Nach der heute vorherrschenden Ansicht läuft die Vermehrung der Viren in der Weise ab, daß zunächst die einzelnen Komponenten der Viruspartikel gebildet werden und diese sich erst danach zu den Viria formieren. Eindeutig nachweisen ließ sich dies u. a. bei den Influenzaviren (vgl. l. c. ${ }^{25}$ ), deren RNS-haltige Innenkomponente (g-Antigen) im Zellkern gebildet wird, während das hämagglutinierende Material ihrer Oberfläche im Cytoplasma erscheint. Beide Viruskomponenten verlagern sich im weiteren Verlauf des Infektionsprozesses in Richtung auf die Zellperipherie, wo sie sich schließlich an der Zellmembran oder in Vorstülpungen derselben zu den reifen Viruspartikeln zusammenschließen. Bei diesem Vorgang werden Bestandteile der Zellmembran mit eingebaut. Die einzelnen Prozesse sind nicht so scharf aufeinander abgestimmt, daß die Wirtszelle bei der Produktion des Virus rationell arbeiten kann. Bei den Influenzaviren werden sowohl das g-Antigen, wie das Hämagglutinin (Viromikrosomen) im Überschuß gebildet und können deshalb nach Abschluß der Virusvermehrung in relativ großer Menge aus den abgestorbenen Zellen isoliert

25 W. SCHÄFER, in: „Virus Growth and Variation“ (eds. A. IsaAcs and B. W. Lacey), University Press, Cambridge 1959. werden. Außerdem können hier Störungen bei der Vereinigung der beiden Komponenten zum reifen Virion auftreten und zwar in der Weise, daß keine oder nur ein Teil der Virusinnenkomponente eingebaut wird (Inkomplette Formen). Nach Untersuchungen mit fluoreszierenden Antikörpern ${ }^{26}$ scheint dies zumindest in den Fällen, in denen hohe Impfdosen verwendet wurden, darauf zu beruhen, daß der Transport der Virusinnenkomponente vom Zellkern zur Zellperipherie gestört ist.

Ebenso wie bei den Influenzaviren scheinen auch beim NDV die einzelnen Prozesse nicht besonders scharf koordiniert zu sein. Der Vermehrungsmechanismus wurde bei diesem Virus zwar noch nicht so eingehend analysiert wie bei den Influenzaviren, es konnte aber inzwischen bereits gezeigt werden, daß auch hier ein RNS-haltiges, virusspezifisches Antigen (NP- bzw. s-Antigen) in den abgestorbenen Wirtszellen enthalten ist, bei dem es sich aller Wahrscheinlichkeit nach um die im Überschuß gebildete Innenkomponente des ND-Virion handelt ${ }^{27}$.

Wie die hier beschriebenen Untersuchungen ergaben, werden darüber hinaus auch beim NDV überschüssige Mengen von Hämagglutinin, das mit Neuraminidase gekoppelt ist, gebildet. Es liegt offenbar im endoplasmatischen Retikulum der Wirtszellen vor, da die entsprechenden isolierten Strukturen (ZGHA) die Eigenschaften von Mikrosomen haben. Sie gleichen diesen elektronenoptisch, enthalten Ribosomen und verhalten sich gegenüber Äther wie die nach Influenzainfektion isolierten, hämagglutinierenden, mikrosomalen Elemente. Es wird vorgeschlagen, sie wie die letzteren als „Viromikrosomen" zu bezeichnen.

Schließlich scheinen analog wie bei den Influenzaviren (Inkomplette Formen) auch beim NDV bei dem letzten Vermehrungsschritt - dem Zusammenbau der beiden Hauptkomponenten zu reifen Viria Störungen auftreten zu können und zwar in der Weise, daß sich wohl deren Hämagglutinin-bewehrte Hülle annähernd normal ausbildet, die Virusinnenkomponente aber nicht inkorporiert wird. Diese Partikel (FHA) werden zumindest beim Stamm „Beaudette" wie die Viria von der Zelle ausgeschieden. Sie verhalten sich gegenüber Äther wie die letzteren und unterscheiden sich in dieser Hinsicht auch nicht von den reifen und inkompletten Partikeln der

${ }^{26}$ R. Rотт, Boerhaave Cursus, Leiden 1960.

27 R. Rott u. I. M. Reda, Zbl. Vet. Med. 10 B, 57 [1963]. 
Influenzaviren. Dies wird als Hinweis dafür gewertet, daß ihre Lipoproteide ursprünglich Bestandteile der peripheren Membran der Wirtszelle waren. Da sie aber die RNS-haltige Innenkomponente nicht enthalten, besitzen sie auch nicht die Fähigkeit, die Bildung neuer Viria zu induzieren. Es erscheint deshalb berechtigt, sie in Analogie zu den entsprechenden Teilchen der Influenzaviren als „Inkomplette Formen" des NDV zu bezeichnen.

Ein Unterschied zu den Influenzaviren besteht insofern, als beim NDV die Infektion mit höheren Virusdosen keinen feststellbaren Einfluß auf die Ausbeute an Inkompletten Formen hat. Dieser Unterschied könnte darauf zurückzuführen sein, daß im Gegensatz zu den Influenzaviren beim NDV sowoh] die Oberflächen- wie die Innen-Komponente des Virion im Cytoplasma fertiggestellt werden; darauf deuten wenigstens die bisher in dieser Richtung mit fluoreszierenden Antikörpern durchgeführten Untersuchungen hin ${ }^{28}$. Es ist leicht einzusehen, daß unter diesen Umständen eine Störung des intrazellulären Transportmechanismus, auf der nach den vorher erwähnten Befunden ${ }^{26}$ der vo n M a g n u s-Effekt der Influenzaviren zu beruhen scheint, sich nicht in entsprechendem Maße auswirken kann.

Unterschiede in der Lokalisation der Virusbildung in der Zelle könnten auch dafür verantwortlich sein, daß bei dem gering-pathogenen ND-Stamm "Beaudette“ mehr Inkomplette Formen als bei dem hoch-pathogenen Stamm "Italien" in die Eiflüssigkeit ausgeschieden werden. Nach den Untersuchungen von $B_{A N G}{ }^{29}$ scheinen die Viria bei den schwachpathogenen ND-Stämmen wie bei den Influenzaviren an der peripheren Zellmembran, bei den hochpathogenen Stämmen dagegen hauptsächlich im Innern des Cytoplasmas fertiggestellt zu werden.

Von den Inkompletten Formen der Influenza unterscheiden sich die der ND noch darin, daß sie keine Interferenz erzeugen. In dieser Hinsicht könnte von Bedeutung sein, daß in den von uns gewonnenen, gereinigten Präparaten von Inkompletten Formen des NDV keine Nucleinsäure nachzuweisen war. Paucker und Henle ${ }^{15}$ haben bereits früher gezeigt, daß der Grad der interferierenden Aktivität der Inkompletten Formen der Influenza von ihrem Gehalt an RNS-haltiger Innenkomponente abhängt.

28 M. I. Traner, R. L. Northrop u. D. L. Walker, Proc. Soc. exp. Biol. Med. 104, 268 [1960].
In diesem Zusammenhang erhebt sich noch die Frage, warum bei den Inkompletten Formen der Influenza häufig noch Teile der Innenkomponente in das Partikel gelangen können, bei den Inkompletten Teilchen der ND diese aber offenbar vollständig fehlt. Dies könnte damit zusammenhängen, daß die RNS-haltige Innenkomponente des NDV verglichen mit der der Influenzaviren relativ stabil ist ${ }^{3,24}$. Im Gegensatz $\mathrm{zu}$ der fragilen Komponente der Influenza-Erreger wird wahrscheinlich die stabilere des NDV entweder in toto oder überhaupt nicht eingebaut.

Die Feststellung, daß Inkomplette Formen nicht nur bei den Influenzaviren, sondern auch bei dem Vertreter einer anderen Virusgruppe auftreten, die zahlreiche medizinisch und veterinärmedizinisch bedeutungsvolle Virusarten zu umfassen scheint, könnte von einer weiterreichenden Bedeutung sein. Gedacht wird dabei an die Entwicklung chemotherapeutischer Verfahren. Sie scheiterte bisher vor allem daran, daß die verwendeten Verbindungen nicht nur die Virusvermehrung, sondern auch den normalen Zellstoffwechsel störten. Auf Grund der hier entwickelten Vorstellung könnte man daran denken, den Infektionsprozeß nicht voll zu unterdrücken, sondern lediglich in der Weise umzusteuern, daß an Stelle von infektiösen Partikeln ausschließlich nichtinfektiöse, aber antigen noch wirksame Inkomplette Formen gebildet werden. Eine solche Umsteuerung erfordert wahrscheinlich nur eine Störung der Transportvorgänge in der Zelle, die nicht von vornherein mit einer Störung der fundamentalen Stoffwechselprozesse verbunden zu sein braucht. Das ergibt sich schon aus der Tatsache, daß Zellsysteme existieren, in denen, obwohl sie keine wesentlichen Abweichungen im Stoffwechsel zeigen, gewisse Transportmechanismen nicht funktionieren. So ist z. B. von den L-Zellen bekannt, daß bei ihnen ohne jede weitere Einwirkung von außen der Transport der Innenkomponente eines Influenzavirus aus dem Kern in das Cytoplasma blockiert und deshalb der Vermehrungszyklus des Virus gestört ist ${ }^{30}$.

Die Untersuchungen wurden durch die D e u t s che F ors chung s gem ein s ch a f $\mathrm{t}$ gefördert. D. KuNert und U. Schäfer danken wir für ihre Mitarbeit.

29 F. B. Bang, Bull. Johns-Hopkins Hosp. 92, 309 [1953].

30 R. M. Franklin u. P. M. Breitenfeld, Virology 8, 293 [1959]. 